\title{
Higher order gravities and the Strong Equivalence Principle
}

\author{
Tomás Ortín \\ Instituto de Física Teórica UAM/CSIC, \\ C/ Nicolás Cabrera, 13-15, C.U. Cantoblanco, E-28049 Madrid, Spain \\ E-mail: Tomas.Ortin@csic.es
}

ABSTRACT: We show that, in all metric theories of gravity with a general covariant action, gravity couples to the gravitational energy-momentum tensor in the same way it couples to the matter energy-momentum tensor order by order in the weak field approximation around flat spacetime. We discuss the relation of this property to the Strong Equivalence Principle. We also study the gauge transformation properties of the gravitational energymomentum tensor.

Keywords: Classical Theories of Gravity, Models of Quantum Gravity

ARXIV EPRINT: 1705.03495 


\section{Contents}

1 Introduction 1

2 Energy-momentum tensors 5

$\begin{array}{lll}3 & \text { Perturbative gravity and gauge invariance } & 6\end{array}$

4 The energy-momentum tensor of higher-order gravity $\quad 7$

$\begin{array}{llr}5 & \text { Extension to higher orders } & 8\end{array}$

6 Gauge transformations of the energy-momentum tensor $\quad 8$

$\begin{array}{llr}7 & \text { Discussion } & 9\end{array}$

\section{Introduction}

Although General Relativity has passed many experimental tests so far, and in spite of the general problems of physical theories of higher order in derivatives, ${ }^{1}$ there are many reasons to consider possible corrections to the Einstein-Hilbert action constructed from invariants of higher order in the Riemann curvature tensor: ${ }^{2}$

1. These are the simplest modifications to General Relativity since they do not require the explicit introduction of other "gravitational fields" (such as scalar fields). The modified gravity theories remain dynamical metric theories, automatically satisfying the Weak Equivalence Principle (WEP) if matter only couples minimally to the metric in the action. ${ }^{3}$ General covariance ensures the on-shell covariant divergenceless of the matter energy-momentum tensor.

2. Most theories of Quantum Gravity (in particular Superstring Theories) predict an infinite number of higher-order corrections to the Einstein-Hilbert action and the effective gravitational theories are higher-order gravity theories, but only the lowest order corrections are explicitly known. In this context, some of the illnesses of the theories with terms of higher order in the curvature can be understood as the result of the truncation of a consistent theory. For Superstring Theories, see, e.g. refs. [5-11]

\footnotetext{
${ }^{1}$ Most of these problems are related to the linear instability discovered by Ostrogradski in refs. [1, 2] and pedagogically reviewed with its implications in ref. [2]. Nevertheless, see also refs. [3, 4].

${ }^{2}$ We are only going to consider metric theories based on a general-covariant action principle.

${ }^{3}$ Field redefinitions of the metric involving the curvature can change this property. We will, henceforth ignore the possibility of making this kind of field redefinitions and we will assume our metric is the one matter would couple minimally to in the action.
} 
3. The AdS/CFT duals of these higher-order gravity theories are much more general than those dual to Einstein-Hilbert gravity and have been proven to be very useful in this context. Some interesting examples can be found in, e.g., refs. [12-20].

4. Typically, the higher-order terms modify the behaviour of the gravitational field in regions of strong curvature. Thus, these corrections may play important rôles in inflationary cosmology, in black-hole physics (see, e.g. refs. [21-33]) and in the study of spacetime singularities.

In this note we are going to call the theories of gravity resulting from the addition of terms of higher order in the Riemann curvature tensor $\mathcal{R}$ or its covariant derivatives $\nabla \mathcal{R}, \nabla^{2} \mathcal{R}, \ldots$ invariant under general coordinate transformations to the Einstein-Hilbert action, that is, theories with actions of the form

$$
S[g]=\frac{1}{\chi^{2}} \int d^{d} x \sqrt{|g|}\{R+F(g, \mathcal{R}, \nabla \mathcal{R}, \cdots)\}, \quad \chi^{2}=16 \pi G,
$$

where $R$ is the Ricci scalar as higher-order gravities. ${ }^{4}$

One of the main obstacles in the study of higher-order gravities is the sheer number of different combinations of curvature invariants that can be constructed as the order in curvature and the dimension grow. For this reason, most of the research has focused on quadratic theories (see, e.g. refs. [11,35-41]) and in the search for theories with special properties [25, 26, 42-47]. Typically, one looks for theories which only propagate, in maximally symmetric vacua, a massless, transverse, graviton or, at the very least, theories that do not propagate the ghost-like spin-2 mode which generically appears in higher-order gravities together with scalar modes [48-50]. Actually, some of the higher-order gravities (the $f(R)[51-53]$ or $f$ (Lovelock) $[54,55]$ theories, for example) can be reformulated as scalar-tensor gravities. In these alternative representations there are issues related to the use of the Jordan or Einstein frames and to the qualification of the scalar-mediated interactions, which are usually different for different kinds of matter, as gravitational or non-gravitational.

Some of these issues can be avoided by sticking to the original, higher-order, metric representation. However, if the theory does propagate other modes besides the massless graviton, one can expect to see some effects associated to them in that representation as well. ${ }^{5}$ One of such effects would be a violation of the Strong Equivalence Principle (SEP) as shown in ref. [56]. The following paragraph in section 3.1.2 of ref. [57] summarizes very well our current understanding: after arguing that only metric theories of gravity have a chance of satisfying the SEP it is stated that

Empirically it has been found that almost every metric theory other than GR introduces auxiliary gravitational fields, either dynamical or prior geometric, and thus predicts violations of SEP at some level (here we ignore quantumtheory inspired modifications to GR involving " $R$ " terms).

\footnotetext{
${ }^{4}$ Observe that purely higher-order gravities (without Einstein-Hilbert term) and Palatini $(f(R)$ or else) theories are excluded from our considerations. For a review of the latter see, e.g. ref. [34].

${ }^{5}$ The existence of other representations for a generic higher-order gravity is, by no means, guaranteed.
} 
The reasons why quantum-theory inspired modifications to GR involving " $R$ " "terms, which are precisely the subject of this note, should be kept out of the discussion are not clear to us. As we have just mentioned, some of them admit a scalar-tensor representation which suggests violations of SEP. On the other hand, there are higher-order theories such as the Einsteinian cubic gravity of ref. [47] which only propagate a transverse, traceless, massless spin-2 mode. Do they violate the SEP? Is it possible to make a general statement concerning the SEP in higher-order gravities?

The difference between the Einstein and the Strong Equivalence Principles (EEP and SEP, resp. $)^{6}$ is that the second extends the former to situations in which the presence of gravitational fields is significant. The main effects of gravitational fields in a local system in free fall are

1. Contributions to the binding energy of macroscopic bodies or Keplerian systems.

2. Determination, through the curvature, of the boundary conditions for locally Minkowskian metrics.

The second effect, which falls out of the scope of this note, is responsible for most of the violations of the SEP in higher-order gravities since, via the curvature of a external gravitational field, free-falling systems can suffer spacetime position-dependent effects. Often they are associated to the emergence of an effective spacetime position-dependent Newton constant, although this concept is far from having a unique and clear definition.

The consequences of the first effect depend on the nature of the coupling of gravity to gravity: the SEP states that it must be identical to the coupling of gravity to other forms of matter/energy so that self-gravitation does not modify the free fall motion of massive bodies. $.^{\prime}, 8$ In metric theories, thus, the SEP demands that the metric couples to the gravitational energy-momentum tensor in the same way it couples to the energymomentum tensor of any other kind of matter. This property only makes sense in the weak-field limit in which a special-relativistic gravitational energy-momentum tensor can be (non-uniquely) defined, but it is a property that any metric theory of gravity can be tested for unambiguously. This is what we are going to do for higher-order gravities.

\footnotetext{
${ }^{6}$ A general formulation of the different Equivalence Principles can be found in, e.g. [58].

7 The necessity of the self-coupling of the relativistic gravitational field arises naturally in the construction of a classical interacting special-relativistic theory of gravity, as reviewed in ref. [59]. The Lorentz invariance of the S matrix of the quantized theory requires the graviton field to couple to the total energy-momentum tensor [60, 61], which , in the long wavelength limit must be that derived from General Relativity in the weak-field limit [62]. At the same time, the consistency of the self-coupling requires the introduction of an infinite number of corrections as first noticed by Gupta [63,64] who devised the possible construction of the full theory by demanding consistency of the self-coupling of the gravitational field at all orders (the socalled "Gupta program"). (Obviously, the meaning of consistency is one of the keys in this problem.) It has been argued that the resulting theory is equivalent to General Relativity [65-68] although this conclusion seems to depend strongly on the starting point, as shown in ref. [69] where a different theory, equivalent to unimodular gravity, was found. Although the theories that we are considering have an Einstein-Hilbert term and, therefore, a Fierz-Pauli term in the weak-field limit (which seems to lead unavoidably to General Relativity) we are not going to use explicitly this fact in what follows and our results will be valid for more general classes of general covariant theories.

${ }^{8}$ The violation of this part of the SEP would give rise to the Nordtvedt effect [70, 71]. Section 2.
} 
Let us start by formulating more precisely this property.

If we expand the gravitational field around the Minkowski spacetime in the weak field approximation $g_{\mu \nu}=\eta_{\mu \nu}+\chi h_{\mu \nu}$ then the SEP requires the equations of motion of the gravitational field to second order in $h_{\mu \nu}$ to have the form ${ }^{9}$

$$
\mathcal{D}^{(0) \mu \nu}=\chi\left(t^{(0) \mu \nu}+T_{\text {matter }}^{\mu \nu}\right),
$$

where $\mathcal{D}^{(0) \mu \nu}$ is a wave operator that acts linearly on $h_{\mu \nu}$ but which is of arbitrary order in derivatives, computed from the quadratic (zeroth-order) term in the action, $t^{(0)} \mu \nu$ is zerothorder gravitational energy-momentum tensor in Minkowski spacetime computed from the same term in the action, quadratic in $h_{\mu \nu}$ but also of arbitrary order in derivatives and $T_{\text {matter }}^{\mu \nu}$ is the matter energy-momentum tensor in Minkowski spacetime computed according to Rosenfeld's prescription [73]. ${ }^{10}$

The consistency of the above equation will be the main ingredient of our discussion. Observe that, if it can be derived from the lowest-order terms of an action ${ }^{11}$

$$
-2 \frac{\delta S}{\delta h_{\mu \nu}}=\mathcal{D}^{(0) \mu \nu}-\chi t^{(0) \mu \nu}+\ldots, \quad S=S^{(0)}+\chi S^{(1)}+\chi^{2} S^{(2)}+\ldots
$$

as we have assumed, the wave operator originates in the variation of the zeroth-order term

$$
\mathcal{D}^{(0) \mu \nu}=-2 \frac{\delta S^{(0)}}{\delta h_{\mu \nu}},
$$

while the gravitational energy-momentum tensor must originate in the first-order term in the action

$$
t^{(0) \mu \nu}=2 \frac{\delta S^{(1)}}{\delta h_{\mu \nu}} .
$$

The variation of $S^{(1)}$ will always give some term quadratic in $h_{\mu \nu}$, but the SEP demands that this term is precisely the energy-momentum tensor of the gravitational field corresponding to $S^{(0)}$.

The rest of this note is devoted to proving that this property, which requires a very precise relation between $S^{(0)}$ and $S^{(1)}$, holds in all higher-order gravities. We will start by recalling some well- and less-well-known facts about special-relativistic energy-momentum tensors of theories of higher-order in derivatives in section 2 . In section 3 we will study the implications of general covariance concerning the gravitational energy-momentum tensor in the context of perturbative gravity and in section 4 we will use them to prove eq. (1.6). Section 7 contains our conclusions and a discussion of how the SEP may be satisfied when terms of higher order in $h_{\mu \nu}$ are considered.

\footnotetext{
${ }^{9}$ Here we follow the notation and conventions of ref. [59]. The consequences of having different couplings for the gravitational and matter energy-momentum tensors $\left(\chi^{\prime}\right.$ and $\left.\chi\right)$ at the linear level were first studied by Kraichnan in ref. [72].

${ }^{10}$ That is: coupling matter minimally to the metric $g_{\mu \nu}$ and computing

$$
T_{\text {matter }}^{\mu \nu}=\left.2 \frac{\delta S_{\text {matter }}}{\delta g_{\mu \nu}}\right|_{g_{\mu \nu}=\eta_{\mu \nu}} .
$$

In the context of the weak-field expansion this is the energy-momentum tensor that occurs naturally coupled to $h_{\mu \nu}$.

${ }^{11}$ We ignore the matter fields from now onwards.
} 


\section{Energy-momentum tensors}

Let us consider a $d$-dimensional field theory with action

$$
S=\int d^{d} x \mathcal{L}
$$

where the Lagrangian $\mathcal{L}$ is a function of the field $\phi$ and its derivatives $\partial_{\mu} \phi, \partial_{\mu} \partial_{\nu} \phi$, $\partial_{\mu} \partial_{\nu} \partial_{\rho} \phi, \ldots$ up to an arbitrary order.

Imposing adequate conditions for the boundary values of the field and a number of its derivatives, demanding the extremization of the above action leads to the Euler-Lagrange equations

$$
\frac{\delta S}{\delta \phi}=\frac{\partial \mathcal{L}}{\partial \phi}-\partial_{\mu} \frac{\partial \mathcal{L}}{\partial \partial_{\mu} \phi}+\partial_{\mu} \partial_{\nu} \frac{\partial \mathcal{L}}{\partial \partial_{\mu} \partial_{\nu} \phi}-\partial_{\mu} \partial_{\nu} \partial_{\rho} \frac{\partial \mathcal{L}}{\partial \partial_{\mu} \partial_{\nu} \partial_{\rho} \phi}+\ldots
$$

According to the first Noether theorem, the invariance of the above action under constant displacements of the coordinates $\delta x^{\mu}$, with $\delta \phi=\delta x^{\mu} \partial_{\mu} \phi$ leads to the relation

$$
\partial_{\mu} t_{\mathrm{can}}{ }^{\mu}{ }_{\nu}=\frac{\delta S}{\delta \phi} \partial_{\nu} \phi
$$

where

$$
t_{\text {can }}{ }^{\mu}{ }_{\nu} \equiv \eta^{\mu}{ }_{\nu} \mathcal{L}-\frac{\partial \mathcal{L}}{\partial \partial_{\mu} \phi} \partial_{\nu} \phi-\frac{\partial \mathcal{L}}{\partial \partial_{\mu} \partial_{\alpha} \phi} \partial_{\nu} \partial_{\alpha} \phi+\partial_{\alpha}\left(\frac{\partial \mathcal{L}}{\partial \partial_{\mu} \partial_{\alpha} \phi}\right) \partial_{\nu} \phi+\ldots
$$

is the canonical energy-momentum tensor. The above relation implies its on-shell conservation (i.e. when $\delta S / \delta \phi=0$ ), but we will need the complete off-shell relation, which, for the gravitational field, at lowest order, takes the form

$$
\partial_{\mu} t_{\mathrm{can} \nu}^{(0) \mu}=\frac{\delta S^{(0)}}{\delta h_{\rho \sigma}} \partial_{\nu} h_{\rho \sigma} .
$$

Noether currents and, in particular, the canonical energy-momentum tensor, are not unambiguously defined: one can add to them terms proportional to the equations of motion, that will vanish on shell, and superpotential terms of the form

$$
\partial_{\rho} \Psi_{\nu}^{\rho \mu}, \text { with } \Psi_{\nu}^{\rho \mu}=\Psi_{\nu}^{[\rho \mu]} .
$$

The positive side of this ambiguity is that it can be used to make gauge-invariant or symmetrize the canonical energy-momentum tensor. Belinfante [74] found a systematic procedure to symmetrize the canonical energy-momentum tensor of fundamental fields that gives the same result as the Rosenfeld prescription for many fields, but not for the gravitational field $h_{\mu \nu}$ unless one adds terms proportional to the equations of motion [59]. In the General Relativity case, it was shown in refs. $[59,75]$ that the gravitational energymomentum tensor which is singled-out by the theory (and satisfies the consistency condition eq. (1.6)) is completely determined by gauge invariance and it is related to the canonical one by a superpotential and on-shell-vanishing terms. It was also shown that using other energy-momentum tensors (as done by Thirring in ref. [76], for instance) leads to the wrong 
value for the secular shift of Mercury's perihelion. The same principle should determine the gravitational energy-momentum tensor in higher-order gravities. ${ }^{12}$ On the other hand, given a conserved 2-index tensor, quadratic in the gravitational field, we will identify it with the energy-momentum tensor if it differs from the canonical one by superpotential and on-shell-vanishing terms.

Let us know examine in more details how gauge invariance acts in this context.

\section{Perturbative gravity and gauge invariance}

Higher-order gravities are covariant under general coordinate transformations $\delta_{\xi} S=0$. In the weak-field limit this symmetry manifests itself order by order in $\chi$

$$
\delta_{\xi}=\delta_{\xi}^{(0)}+\chi \delta_{\xi}^{(1)}+\chi^{2} \delta_{\xi}^{(2)}+\ldots
$$

The variation of the gravitational fields $h_{\mu \nu}$, only has zeroth- and first-order terms: zeroth- and first-order transformations

$$
\delta_{\xi} h_{\mu \nu}=\left(\delta_{\xi}^{(0)}+\chi \delta_{\xi}^{(1)}\right) h_{\mu \nu},
$$

with

$$
\delta_{\xi}^{(0)} h_{\mu \nu}=2 \partial_{(\mu} \xi_{\nu)}, \quad \delta_{\xi}^{(1)} h_{\mu \nu}=£_{\xi} h_{\mu \nu}=\xi^{\rho} \partial_{\rho} h_{\mu \nu}+2 \partial_{(\mu} \xi^{\rho} h_{\nu) \rho}
$$

The parameter $\xi^{\mu}$ is completely arbitrary and it does not satisfy any constraints restricting the general covariance of the original theory. This condition excludes the unimodular theories which are invariant under the above transformations for divergenceless parameters $\partial_{\mu} \xi^{\mu}=0$ only [77].

These transformations relate terms of consecutive orders in the action:

$$
\begin{aligned}
\delta_{\xi}^{(0)} S^{(0)} & =0, \\
\delta_{\xi}^{(0)} S^{(n)}+\delta_{\xi}^{(1)} S^{(n-1)} & =0, \text { for } n \leq 1 .
\end{aligned}
$$

To our purposes, each of these relations can be seen as an independent gauge symmetry, and there is a Noether (also called gauge, or Bianchi) identity associated to each of these local symmetries via Noether's second theorem. Using the explicit form of the transformations of the gravitational field one arrives, after integration by parts and elimination

\footnotetext{
${ }^{12}$ It should also determine the higher-order corrections for Fierz-Pauli that eventually lead to General Relativity. In the search for theories with consistent self-coupling of the gravitational field $h_{\mu \nu}$ (Gupta's program) mentioned in footnote 7 , if one just tries to couple the gravitational field to its own energymomentum tensor, the ambiguity in the definition of the latter turns the problem into the problem of which is the "right" energy-momentum tensor. Even if one can show that there is a prescription that in the end gives General Relativity, as in ref. [67], one needs some physical principle to justify it and its uniqueness. Gauge invariance plays here this rôle.
} 
of total derivatives, to the first two Noether identities ${ }^{13}$

$$
\begin{aligned}
& \partial_{\mu} \frac{\delta S^{(0)}}{\delta h_{\mu \nu}}=0 \\
& \partial_{\mu} \frac{\delta S^{(1)}}{\delta h_{\mu \nu}}=-\partial_{\mu}\left(\frac{\delta S^{(0)}}{\delta h_{\mu \rho}} h_{\rho}^{\nu}\right)+\frac{1}{2} \frac{\delta S^{(0)}}{\delta h_{\rho \sigma}} \partial^{\nu} h_{\rho \sigma} .
\end{aligned}
$$

Together, if eq. (1.6) is satisfied, they are consistent with the on-shell conservation of the gravitational energy-momentum tensor to lowest order in $\chi$ : if we take the divergence of both sides of the equation of motion (1.2) and use the first identity, we get

$$
\chi \partial_{\mu} t^{(0) \mu \nu}=0 .
$$

The second identity says that

$$
\chi \partial_{\mu} t^{(0) \mu \nu}=-2 \chi \partial_{\mu}\left(\frac{\delta S^{(0)}}{\delta h_{\mu \rho}} h_{\rho}^{\nu}\right)+\chi \frac{\delta S^{(0)}}{\delta h_{\rho \sigma}} \partial^{\nu} h_{\rho \sigma},
$$

and, using again the equations of motion

$$
\chi \partial_{\mu} t^{(0) \mu \nu}=\mathcal{O}\left(\chi^{2}\right), \quad \text { on shell. }
$$

Apart from this consistency check, let us stress that the above identities eqs. (3.6), (3.7) hold off-shell for any higher-order gravity.

\section{The energy-momentum tensor of higher-order gravity}

We are now ready to prove that eq. (1.6) holds; that is: that $2 \delta S^{(1)} / \delta h_{\mu \nu}$ can be identified with one of the special-relativistic energy-momentum tensors that one can associate to $S^{(0)}$, such as the canonical energy-momentum tensor $t_{\mathrm{can}}^{(0)}{ }_{\nu}{ }_{\nu}$ plus some on-shell-vanishing terms (to lowest order in $\chi$ !) and a superpotential term.

Using in the Noether identity eq. (3.7) the property eq. (2.5) we can rewrite it as follows:

$$
\partial_{\mu}\left(2 \frac{\delta S^{(1)}}{\delta h_{\mu \nu}}\right)=\partial_{\mu}\left(t_{\text {can }}^{(0) \mu \nu}-2 \frac{\delta S^{(0)}}{\delta h_{\mu \rho}} h_{\rho}^{\nu}\right),
$$

from which it follows that

$$
2 \frac{\delta S^{(1)}}{\delta h_{\mu \nu}}=t_{\mathrm{can}}^{(0) \mu \nu}-2 \frac{\delta S^{(0)}}{\delta h_{\mu \rho}} h_{\rho}^{\nu}+\partial_{\rho} \Psi^{(0) \rho \mu \nu}, \quad \text { with } \quad \Psi^{(0) \rho \mu \nu}=-\Psi^{(0) \mu \rho \nu} .
$$

Given that the second term in the r.h.s. vanishes on-shell at the order in $\chi$ we are working at, ${ }^{14}$ the l.h.s. of the above equation can be identified with a special-relativistic gravitational energy-momentum tensor associated to the zeroth-order action $S^{(0)}$, proving eq. (1.6).

\footnotetext{
13 The restricted gauge invariance of linearized unimodular gravity leads to different Noether identities [78, 79]. The classical equations of motion of unimodular gravity [80] are those of General Relativity with a cosmological constant and, presumably, this theory must enjoy the same property we are proving here, although it must arise in a more complicated way.

${ }^{14}$ Equivalently, if we use the equations of motion, that term would be of $\mathcal{O}\left(h^{3}\right)$.
} 


\section{$5 \quad$ Extension to higher orders}

Can we extend this $\mathcal{O}(h)$ result to higher orders?

The $n$th Noether identity is

$$
\partial_{\mu} \frac{\delta S^{(n)}}{\delta h_{\mu \nu}}=-\partial_{\mu}\left(\frac{\delta S^{(n-1)}}{\delta h_{\mu \rho}} h_{\rho}^{\nu}\right)+\frac{1}{2} \frac{\delta S^{(n-1)}}{\delta h_{\rho \sigma}} \partial^{\nu} h_{\rho \sigma},
$$

and using again eq. (2.5) and the same reasoning as in the $n=1$ case, we arrive to

$$
2 \frac{\delta S^{(n)}}{\delta h_{\mu \nu}}=t_{\mathrm{can}}^{(n-1) \mu \nu}-2 \frac{\delta S^{(n-1)}}{\delta h_{\mu \rho}} h_{\rho}^{\nu}+\partial_{\rho} \Psi^{(n-1) \rho \mu \nu}, \quad \text { with } \quad \Psi^{(n-1) \rho \mu \nu}=-\Psi^{(n-1) \mu \rho \nu} .
$$

where $t_{\text {can }}^{(n-1)} \mu \nu$ is the contribution to the gravitational special-relativistic canonical energymomentum tensor coming from the $S^{(n-1)}$. Adding all these relations to order $n$ multiplied by the corresponding power of $\chi$ we get

$$
\begin{aligned}
2\left(\chi \frac{\delta S^{(1)}}{\delta h_{\mu \nu}}+\chi^{2} \frac{\delta S^{(2)}}{\delta h_{\mu \nu}}+\cdots+\chi^{n} \frac{\delta S^{(n)}}{\delta h_{\mu \nu}}\right)= & \chi t_{\mathrm{can}}^{(0) \mu \nu}+\chi^{2} t_{\mathrm{can}}^{(1) \mu \nu}+\cdots+\chi^{n} t_{\mathrm{can}}^{(n-1) \mu \nu} \\
& -2 \chi\left(\frac{\delta S^{(0)}}{\delta h_{\mu \rho}}+\chi \frac{\delta S^{(1)}}{\delta h_{\mu \rho}}+\cdots+\chi^{n-1} \frac{\delta S^{(n-1)}}{\delta h_{\mu \rho}}\right) h_{\rho}^{\nu} \\
& +\partial_{\rho}\left(\chi \Psi^{(0) \rho \mu \nu}+\chi^{2} \Psi^{(1) \rho \mu \nu}+\cdots++\chi^{n} \Psi^{(n-1) \rho \mu \nu}\right) .
\end{aligned}
$$

The $n$ th-order equations of motion say that

$$
\frac{\delta S^{(0)}}{\delta h_{\mu \nu}}+\chi \frac{\delta S^{(1)}}{\delta h_{\mu \nu}}+\cdots+\chi^{n-1} \frac{\delta S^{(n-1)}}{\delta h_{\mu \nu}}=-\chi^{n} \frac{\delta S^{(n)}}{\delta h_{\mu \nu}}
$$

and, therefore, the term in the second line of the r.h.s. of eq. (5.3) vanishes up to this order on-shell and the term in the l.h.s. can be identified with a special-relativistic energymomentum tensor for the gravitational field.

\section{Gauge transformations of the energy-momentum tensor}

The lowest-order energy-momentum tensor of the spin-2 field, $t_{\mathrm{GR} \mu \nu}^{(0)}$ is not expected to be invariant under the $\delta_{\xi}^{(0)}$ gauge transformations. Its gauge transformation rule must be completely determined by the gauge-invariance of the action and the same must be true for $t_{\mu \nu}^{(0)}$ and its higher order in $h$ corrections in higher-order theories. In order to find this gauge transformation rule we proceed as follows: we first compute the gauge trasformations eqs. (3.2) and (3.3) of the action $\delta_{\xi} S[h]$ order by order in $\chi$. By assumption $\delta_{\xi} S[h]=0$ up to total erivatives. Then we take another general variation with respect to $h_{\mu \nu}, \delta \delta_{\xi} S[h]=0$, taking into account that $\frac{\delta \delta_{\xi}^{(0)} h_{\mu \nu}}{\delta h_{\alpha \beta}}=0$ but $\frac{\delta \delta_{\xi}^{(1)} h_{\mu \nu}}{\delta h_{\alpha \beta}} \neq 0$, so that, integrating by parts

$$
\int d^{d} x \frac{\delta S^{(n)}}{\delta h_{\mu \nu}} \frac{\delta \delta_{\xi}^{(1)} h_{\mu \nu}}{\delta h_{\alpha \beta}}=\int d^{d} x\left\{-\partial_{\rho}\left(\xi^{\rho} \frac{\delta S^{(n)}}{\delta h_{\alpha \beta}}\right)+2 \partial^{(\alpha} \xi_{\rho} \frac{\delta S^{(n)}}{\delta h_{\beta) \rho}}\right\} .
$$


Next, we interchange the variations of the $S^{(n)}$, arriving to

$$
\begin{aligned}
\delta \delta_{\xi} S[h]=\int d^{d} x\left\{\delta_{\xi}^{(0)} \frac{\delta S^{(0)}}{\delta h_{\alpha \beta}}+\chi\right. & {\left[\delta_{\xi}^{(1)} \frac{\delta S^{(0)}}{\delta h_{\alpha \beta}}+\delta_{\xi}^{(0)} \frac{\delta S^{(1)}}{\delta h_{\alpha \beta}}-\partial_{\rho}\left(\xi^{\rho} \frac{\delta S^{(0)}}{\delta h_{\alpha \beta}}\right)\right.} \\
& \left.\left.+2 \partial^{(\alpha} \xi_{\rho} \frac{\delta S^{(0)}}{\delta h_{\beta) \rho}}\right]+\cdots\right\} \delta h_{\alpha \beta}=0 .
\end{aligned}
$$

Finally, the standard arguments lead to the identities

$$
\delta_{\xi}^{(0)} \frac{\delta S^{(n)}}{\delta h_{\alpha \beta}}=-\delta_{\xi}^{(1)} \frac{\delta S^{(n-1)}}{\delta h_{\alpha \beta}}+\partial_{\rho}\left(\xi^{\rho} \frac{\delta S^{(n-1)}}{\delta h_{\alpha \beta}}\right)-2 \partial^{(\alpha} \xi_{\rho} \frac{\delta S^{(n-1)}}{\delta h_{\beta) \rho}}, \quad \forall n \geq 0 .
$$

Obviously, the sums of the terms $n=1, \cdots N$ and those of the terms $n=0, \cdots N-1$ satisfy the same relation.

For GR at lowest order, these identities imply

$$
\begin{aligned}
\delta_{\xi}^{(0)} \mathcal{D}^{(0) \mu \nu} & =0 \\
\delta_{\xi}^{(0)} t_{\mathrm{GR}}^{(0) \mu \nu} & =\delta_{\xi}^{(1)} \mathcal{D}^{(0) \mu \nu}+\partial_{\rho}\left(\xi^{\rho} \mathcal{D}^{(0) \mu \nu}\right)-2 \partial^{(\mu \mid} \xi_{\rho} \mathcal{D}^{(0) \mid \nu) \rho},
\end{aligned}
$$

which can be checked to hold using the Fierz-Pauli equation of motion and the explicit expression for $t_{\mathrm{GR}}^{(0) \mu \nu}$ in eq. (3.200) of ref. [59]. The equation

$$
\mathcal{D}^{(0) \mu \nu}=\chi t_{\mathrm{GR}}^{(0) \mu \nu},
$$

is only invariant under $\left(\delta_{\xi}^{(0)}+\chi \delta_{\xi}^{(1)}\right) h_{\mu \nu}$ and only up to terms proportional to $\mathcal{D}^{(0) \mu \nu}$ and its derivatives which, on-shell, are of order $\mathcal{O}\left(\chi^{2}\right)$.

\section{Discussion}

In this note we have shown how general covariance in metric theories leads to equations of motion for the perturbative gravitational field sourced by the gravitational energymomentum tensor computed to any order.

As we have stressed in the introduction, this result shows that in all the theories under consideration the gravitational binding energy of massive bodies will not modify their freefall motion, which is one of the requirements of the SEP. Violations of other requirements of the SEP are generally expected and our result has nothing to say about them. It can be said that we have only shown a "microscopic version of the SEP."

This result has nothing to say about the correctness of the theories, either. It only shows that they are internally consistent and that this is due to the gauge invariance that follows from general covariance.

Our result also raises the possibility of carrying out Gupta's program starting with linearized theories of higher order in derivatives at the evel of the actin or at the level of the equations of motion (section 6). Each correction is strongly constrained by the requirement of gauge invariance, suggesting that there is a unique correction at each order 
and that, the whole theory is completely determined by the lowest order that describes the free one.

As discussed in footnote 13, it would also be very interesting to understand if and how the unimodular theory of gravity (and its higher-order generalizations) enjoy similar properties to understand to which extent general covariance is fundamental. Other extensions of this work that would interesting to study are the analysis in De Sitter and anti-De Sitter backgrounds for which selfconsitency is known to lead to GR plus a cosmological constant [81].

\section{Acknowledgments}

The author would like to thank E. Álvarez, F. Barbero, P. Bueno, P.A. Cano, R. CarballoRubio, S. Deser, R. Emparan, L.J. Garay, G.J. Olmo and J.M.M. Senovilla for many interesting and useful comments on this topic and on the draft. He would also like to express a special thanks to the Mainz Institute for Theoretical Physics (MITP) for its hospitality and support and M.M. Fernández for her permanent support. This work has been supported in part by the MINECO/FEDER, UE grant FPA2015-66793-P and the Centro de Excelencia Severo Ochoa Program grant SEV-2012-0249.

Open Access. This article is distributed under the terms of the Creative Commons Attribution License (CC-BY 4.0), which permits any use, distribution and reproduction in any medium, provided the original author(s) and source are credited.

\section{References}

[1] M. Ostrogradski, Les equations differentielles, Mem. Ac. St. Petersbourg VI 4 (1850) 385.

[2] R.P. Woodard, Ostrogradsky's theorem on Hamiltonian instability, Scholarpedia 10 (2015) 32243 [arXiv: 1506.02210] [INSPIRE].

[3] M. Pavšič, Stable Self-Interacting Pais-Uhlenbeck Oscillator, Mod. Phys. Lett. A 28 (2013) 1350165 [arXiv: 1302 .5257] [INSPIRE].

[4] M. Pavšič, Pais-Uhlenbeck Oscillator with a Benign Friction Force, Phys. Rev. D 87 (2013) 107502 [arXiv: 1304.1325] [INSPIRE].

[5] D.J. Gross and E. Witten, Superstring Modifications of Einstein's Equations, Nucl. Phys. B 277 (1986) 1 [INSPIRE].

[6] M.T. Grisaru and D. Zanon, $\sigma$ Model Superstring Corrections to the Einstein-hilbert Action, Phys. Lett. B 177 (1986) 347 [INSPIRE].

[7] M.T. Grisaru, A.E.M. van de Ven and D. Zanon, Two-Dimensional Supersymmetric $\sigma$-models on Ricci Flat Kähler Manifolds Are Not Finite, Nucl. Phys. B 277 (1986) 388 [InSPIRE].

[8] M.T. Grisaru, A.E.M. van de Ven and D. Zanon, Four Loop Divergences for the $N=1$ Supersymmetric Nonlinear $\sigma$-model in Two-Dimensions, Nucl. Phys. B 277 (1986) 409 [INSPIRE].

[9] D.J. Gross and J.H. Sloan, The Quartic Effective Action for the Heterotic String, Nucl. Phys. B 291 (1987) 41 [INSPIRE]. 
[10] E.A. Bergshoeff and M. de Roo, The Quartic Effective Action of the Heterotic String and Supersymmetry, Nucl. Phys. B 328 (1989) 439 [INSPIRE].

[11] L. Álvarez-Gaumé, A. Kehagias, C. Kounnas, D. Lüst and A. Riotto, Aspects of Quadratic Gravity, Fortsch. Phys. 64 (2016) 176 [arXiv: 1505.07657] [INSPIRE].

[12] M. Brigante, H. Liu, R.C. Myers, S. Shenker and S. Yaida, Viscosity Bound Violation in Higher Derivative Gravity, Phys. Rev. D 77 (2008) 126006 [arXiv:0712.0805] [INSPIRE].

[13] A. Buchel and R.C. Myers, Causality of Holographic Hydrodynamics, JHEP 08 (2009) 016 [arXiv:0906.2922] [INSPIRE].

[14] X.O. Camanho and J.D. Edelstein, Causality in AdS/CFT and Lovelock theory, JHEP 06 (2010) 099 [arXiv:0912.1944] [INSPIRE].

[15] R.-G. Cai, Z.-Y. Nie, N. Ohta and Y.-W. Sun, Shear Viscosity from Gauss-Bonnet Gravity with a Dilaton Coupling, Phys. Rev. D 79 (2009) 066004 [arXiv:0901.1421] [INSPIRE].

[16] A. Buchel, J. Escobedo, R.C. Myers, M.F. Paulos, A. Sinha and M. Smolkin, Holographic GB gravity in arbitrary dimensions, JHEP 03 (2010) 111 [arXiv:0911.4257] [INSPIRE].

[17] R.C. Myers, M.F. Paulos and A. Sinha, Holographic studies of quasi-topological gravity, JHEP 08 (2010) 035 [arXiv: 1004.2055] [INSPIRE].

[18] R.C. Myers and A. Sinha, Seeing a c-theorem with holography, Phys. Rev. D 82 (2010) 046006 [arXiv: 1006 .1263] [INSPIRE].

[19] M. Mezei, Entanglement entropy across a deformed sphere, Phys. Rev. D 91 (2015) 045038 [arXiv: 1411.7011] [INSPIRE].

[20] P. Bueno, R.C. Myers and W. Witczak-Krempa, Universality of corner entanglement in conformal field theories, Phys. Rev. Lett. 115 (2015) 021602 [arXiv:1505.04804] [INSPIRE].

[21] D.G. Boulware and S. Deser, String Generated Gravity Models, Phys. Rev. Lett. 55 (1985) 2656 [INSPIRE].

[22] J.T. Wheeler, Symmetric Solutions to the Maximally Gauss-Bonnet Extended Einstein Equations, Nucl. Phys. B 273 (1986) 732 [inSPIRE].

[23] K. Behrndt, G. Lopes Cardoso, B. de Wit, D. Lüst, T. Mohaupt and W.A. Sabra, Higher order black hole solutions in $N=2$ supergravity and Calabi-Yau string backgrounds, Phys. Lett. B 429 (1998) 289 [hep-th/9801081] [INSPIRE].

[24] R.-G. Cai, Gauss-Bonnet black holes in AdS spaces, Phys. Rev. D 65 (2002) 084014 [hep-th/0109133] [INSPIRE].

[25] J. Oliva and S. Ray, A new cubic theory of gravity in five dimensions: Black hole, Birkhoff's theorem and C-function, Class. Quant. Grav. 27 (2010) 225002 [arXiv:1003.4773] [INSPIRE].

[26] R.C. Myers and B. Robinson, Black Holes in Quasi-topological Gravity, JHEP 08 (2010) 067 [arXiv: 1003.5357] [INSPIRE].

[27] H. Lü, A. Perkins, C.N. Pope and K.S. Stelle, Black Holes in Higher-Derivative Gravity, Phys. Rev. Lett. 114 (2015) 171601 [arXiv:1502.01028] [INSPIRE].

[28] A. Kehagias, C. Kounnas, D. Lüst and A. Riotto, Black hole solutions in $R^{2}$ gravity, JHEP 05 (2015) 143 [arXiv: 1502.04192] [INSPIRE]. 
[29] S.H. Hendi, S. Panahiyan and B. Eslam Panah, Magnetic branes in Gauss-Bonnet gravity with nonlinear electrodynamics: correction of magnetic branes in Einstein-Maxwell gravity, Eur. Phys. J. C 75 (2015) 296 [arXiv:1506.02481] [INSPIRE].

[30] R.A. Hennigar and R.B. Mann, Black holes in Einsteinian cubic gravity, Phys. Rev. D 95 (2017) 064055 [arXiv: 1610.06675] [INSPIRE].

[31] P. Bueno and P.A. Cano, Four-dimensional black holes in Einsteinian cubic gravity, Phys. Rev. D 94 (2016) 124051 [arXiv: 1610.08019] [InSPIRE].

[32] R.A. Hennigar, D. Kubizňák and R.B. Mann, Generalized quasitopological gravity, Phys. Rev. D 95 (2017) 104042 [arXiv: 1703.01631] [INSPIRE].

[33] P. Bueno and P.A. Cano, On black holes in higher-derivative gravities, Class. Quant. Grav. 34 (2017) 175008 [arXiv: 1703.04625] [INSPIRE].

[34] G.J. Olmo, Palatini Approach to Modified Gravity: $f(R)$ Theories and Beyond, Int. J. Mod. Phys. D 20 (2011) 413 [arXiv:1101.3864] [INSPIRE].

[35] K.S. Stelle, Renormalization of Higher Derivative Quantum Gravity, Phys. Rev. D 16 (1977) 953 [INSPIRE].

[36] K.S. Stelle, Classical Gravity with Higher Derivatives, Gen. Rel. Grav. 9 (1978) 353 [INSPIRE].

[37] J. Julve and M. Tonin, Quantum Gravity with Higher Derivative Terms, Nuovo Cim. B 46 (1978) 137 [INSPIRE].

[38] D.G. Boulware, G.T. Horowitz and A. Strominger, Zero Energy Theorem for Scale Invariant Gravity, Phys. Rev. Lett. 50 (1983) 1726 [InSPIRE].

[39] F. David and A. Strominger, On the Calculability of Newton's Constant and the Renormalizability of Scale Invariant Quantum Gravity, Phys. Lett. B 143 (1984) 125 [INSPIRE].

[40] G.T. Horowitz, Quantum Cosmology With a Positive Definite Action, Phys. Rev. D 31 (1985) 1169 [INSPIRE].

[41] S. Deser and B. Tekin, New energy definition for higher curvature gravities, Phys. Rev. D 75 (2007) 084032 [gr-qc/0701140] [INSPIRE].

[42] D. Lovelock, Divergence-free tensorial concomitants, Aequationes Math. 4 (1970) 127.

[43] D. Lovelock, The Einstein tensor and its generalizations, J. Math. Phys. 12 (1971) 498 [INSPIRE].

[44] E.A. Bergshoeff, O. Hohm and P.K. Townsend, Massive Gravity in Three Dimensions, Phys. Rev. Lett. 102 (2009) 201301 [arXiv:0901.1766] [INSPIRE].

[45] H. Lü and C.N. Pope, Critical Gravity in Four Dimensions, Phys. Rev. Lett. 106 (2011) 181302 [arXiv: 1101.1971] [INSPIRE].

[46] A. Karasu, E. Kenar and B. Tekin, Minimal extension of Einstein's theory: The quartic gravity, Phys. Rev. D 93 (2016) 084040 [arXiv: 1602.02567] [InSPIRE].

[47] P. Bueno and P.A. Cano, Einsteinian cubic gravity, Phys. Rev. D 94 (2016) 104005 [arXiv: 1607.06463] [INSPIRE].

[48] T.C. Sisman, I. Gullu and B. Tekin, All unitary cubic curvature gravities in D dimensions, Class. Quant. Grav. 28 (2011) 195004 [arXiv:1103.2307] [INSPIRE]. 
[49] B. Tekin, Particle Content of Quadratic and $f\left(R_{\mu \nu \sigma \rho}\right)$ Theories in $(A) d S$, Phys. Rev. D 93 (2016) 101502 [arXiv: 1604.00891] [InSPIRE].

[50] P. Bueno, P.A. Cano, V.S. Min and M.R. Visser, Aspects of general higher-order gravities, Phys. Rev. D 95 (2017) 044010 [arXiv:1610.08519] [InSPIRE].

[51] T.P. Sotiriou and V. Faraoni, f(R) Theories Of Gravity, Rev. Mod. Phys. 82 (2010) 451 [arXiv:0805.1726] [INSPIRE].

[52] A. De Felice and S. Tsujikawa, $f(R)$ theories, Living Rev. Rel. 13 (2010) 3 [arXiv: 1002.4928] [INSPIRE].

[53] S. Nojiri and S.D. Odintsov, Unified cosmic history in modified gravity: from $F(R)$ theory to Lorentz non-invariant models, Phys. Rept. 505 (2011) 59 [arXiv: 1011. 0544] [INSPIRE].

[54] S. Sarkar and A.C. Wall, Generalized second law at linear order for actions that are functions of Lovelock densities, Phys. Rev. D 88 (2013) 044017 [arXiv:1306.1623] [INSPIRE].

[55] P. Bueno, P.A. Cano, A.O. Lasso and P.F. Ramírez, f(Lovelock) theories of gravity, JHEP 04 (2016) 028 [arXiv: 1602.07310] [INSPIRE].

[56] B. Bertotti and L.P. Grishchuk, The strong equivalence principle, Class. Quant. Grav. 7 (1990) 1733.

[57] C.M. Will, The Confrontation between General Relativity and Experiment, Living Rev. Rel. 17 (2014) 4 [arXiv: 1403.7377] [INSPIRE].

[58] E. Di Casola, S. Liberati and S. Sonego, Nonequivalence of equivalence principles, Am. J. Phys. 83 (2015) 39 [arXiv:1310.7426] [INSPIRE].

[59] T. Ortín, Gravity and Strings, second edition, Cambridge University Press (2015).

[60] S. Weinberg, Photons and Gravitons in s Matrix Theory: Derivation of Charge Conservation and Equality of Gravitational and Inertial Mass, Phys. Rev. 135 (1964) B1049 [INSPIRE].

[61] S. Weinberg, Derivation of Gauge Invariance and the Equivalence Principle from Lorentz Invariance in the S Matrix, Phys. Lett. 9 (1964) 357.

[62] D.G. Boulware and S. Deser, Classical General Relativity Derived from Quantum Gravity, Annals Phys. 89 (1975) 193 [INSPIRE].

[63] S.N. Gupta, Gravitation and Electromagnetism, Phys. Rev. 96 (1954) 1683 [InSPIRE].

[64] S.N. Gupta, Einstein's and Other Theories of Gravitation, Rev. Mod. Phys. 29 (1957) 334.

[65] R.H. Kraichnan, Special-Relativistic Derivation of Generally Covariant Gravitation Theory, Phys. Rev. 98 (1955) 1118 [inSPIRE].

[66] S. Deser, Selfinteraction and gauge invariance, Gen. Rel. Grav. 1 (1970) 9 [gr-qc/0411023] [INSPIRE].

[67] L.M. Butcher, M. Hobson and A. Lasenby, Bootstrapping gravity: A consistent approach to energy-momentum self-coupling, Phys. Rev. D 80 (2009) 084014 [arXiv:0906.0926] [INSPIRE].

[68] S. Deser, Gravity from self-interaction redux, Gen. Rel. Grav. 42 (2010) 641 [arXiv: 0910.2975] [INSPIRE].

[69] C. Barceló, R. Carballo-Rubio and L.J. Garay, Unimodular gravity and general relativity from graviton self-interactions, Phys. Rev. D 89 (2014) 124019 [arXiv:1401.2941] [INSPIRE]. 
[70] K. Nordtvedt, Equivalence Principle for Massive Bodies. 1. Phenomenology, Phys. Rev. 169 (1968) 1014 [INSPIRE].

[71] K. Nordtvedt, Equivalence Principle for Massive Bodies. 2. Theory, Phys. Rev. 169 (1968) 1017 [INSPIRE].

[72] R.H. Kraichnan, Possibility of Unequal Gravitational and Inertial Masses, Phys. Rev. 107 (1957) 1485.

[73] L. Rosenfeld, Sur le tenseur d'impulsion-énergie, Mém. Acad. Roy. Belgique 6 (1930) 30.

[74] F.J. Belinfante, On the Spin Angular Momentum of Mesons, Physica VI (1939) 887.

[75] R.P. Feynman, F.B. Morinigo, W.G. Wagner and B. Hatfield, Feynman lectures on gravitation, Addison-Wesley, Reading, U.S.A. (1995).

[76] W.E. Thirring, An alternative approach to the theory of gravitation, Annals Phys. 16 (1961) 96 [INSPIRE].

[77] M. Kreuzer, Gauge Theory of Volume Preserving Diffeomorphisms, Class. Quant. Grav. 7 (1990) 1303 [INSPIRE].

[78] E. Álvarez, Can one tell Einstein's unimodular theory from Einstein's general relativity?, JHEP 03 (2005) 002 [hep-th/0501146] [INSPIRE].

[79] E. Álvarez, D. Blas, J. Garriga and E. Verdaguer, Transverse Fierz-Pauli symmetry, Nucl. Phys. B 756 (2006) 148 [hep-th/0606019] [INSPIRE].

[80] A. Einstein, Do gravitational fields play an essential part in the structure of the elementary particles of matter?, Sitzungsber. Preuss. Akad. Wiss. Berlin (Math. Phys.) 1919 (1919) 433. English translation in A. Einstein et al., The principle of relativity, Dover.

[81] S. Deser, Gravity From Selfinteraction in a Curved Background, Class. Quant. Grav. 4 (1987) L99 [inSPIRE]. 